\title{
LIQUID SLOSHING IN CYLINDRICAL FUEL TANKS
}

\section{E. J. Hopfinger and V. Baumbach}

\begin{abstract}
Results are presented on asymmetric sloshing in partially filled circular, cylindrical tanks subjected to horizontal, periodic forcing, as well as on geyser formation and following sloshing caused by a sudden change in Bond number. Asymmetric sloshing is controlled by three parameters that are the liquid depth parameter, the frequency offset parameter (depending on forcing frequency and forcing amplitude), and the damping. Results are presented that cover a wide range of forcing frequencies and forcing amplitudes in the asymptotic limit of large fluid depth and low viscosity liquids. Geyser formation and axisymmetric sloshing is initiated by dam-break experiments, simulating a sudden change in Bond number. This problem is encountered in liquid-propellant fuel tanks where under microgravity conditions, the liquid rises near the container wall. Engine restart (sudden axial acceleration) can cause geyser formation followed by violent liquid sloshing and gas entrainment.
\end{abstract}

\section{INTRODUCTION}

Liquid sloshing is frequently encountered in liquid storage tanks subjected to external excitations. The forces exerted on the container walls by the sloshing motions and possible large pressure changes caused by enhanced evaporation or condensation at the liquid-gas interface can lead to accidents. Sloshing is also an interesting fundamental problem, related with nonlinear oscillators and dynamic systems $[1,2]$. The book of Ibrahim [3] gives a detailed summary of the theory and fundamentals of sloshing under widely different conditions and contains approximately 3,000 references. Abramson in his foreword to this book mentions that nonlinear sloshing can be bewildering in its complexity. During the early developments of space flights, liquid sloshing in fuel tanks has received considerable attention. A collection of this research can be found in Abramson [4]. In this volume, the sloshing wave modes have been analyzed in detail for containers of various geometries, including nonlinear sloshing and geometric effects on the damping of the liquid motion. The fluid depth is an important parameter because resonant waves have negative or positive nonlinearity, depending on the liquid depth relative to the tank base dimension [5-7]. An interesting aspect is

This is an Open Access article distributed under the terms of the Creative Commons Attribution-Noncommercial License 3.0, which permits unrestricted use, distribution, and reproduction in any noncommercial medium, provided the original work is properly cited. 
that in circular, cylindrical tanks, as well as in square-base cylindrical containers, large amplitude asymmetric planar waves bifurcate to a swirling wave motion at forcing frequencies near and above the natural frequency depending on forcing amplitude and fluid depth $[1,4,7,8]$.

Most of the time, the containers are subjected to lateral forcing giving rise to asymmetric gravity wave motions. Forcing, parallel to the container axis and impulsive forcing is also encountered in rocket engine fuel tanks during engine restart after a ballistic phase. This can lead to geyser formation and subsequent symmetric sloshing. Membranes, simulating high surface tension, have been used to simulate a ballistic phase [9]; rupture of the membrane then leads to a sudden increase in Bond number. In the present experiments, a rigid barrier (infinite surface tension) instead of a membrane has been used to simulate low, initial Bond number conditions.

First, experimental results on large amplitude asymmetric sloshing and wave breaking for large fluid depths, $h / R>1.5$, are presented in section 2 . Then, in section 3 , the dam-break experiments, simulating impulsive forcing leading to geyser formation and axisymmetric sloshing, are discussed.

\section{LATERAL FORCING EXPERIMENTS}

\subsection{Theoretical Concepts}

In order to upscale the results obtained in tanks of laboratory size to large tanks, it is necessary to determine the relevant control or similarity parameters. For a circular, cylindrical tank, subjected to horizontal forcing of tank displacement $x=A_{f} \sin \omega t$, Miles' weakly nonlinear theory [1] contains three parameters (the Bond number is assumed to be large so that surface tension effects are negligible on the tank scale). These parameters are the frequency offset $\beta$, the length $l / R$ parameter, and the damping $\alpha$. For the lowest asymmetric wave mode of natural frequency $\omega_{1}$, these nondimensional parameters are, respectively,

$$
\begin{aligned}
\beta & =\frac{\omega^{2}-\omega_{1}^{2}}{\varepsilon^{2} \omega_{1}^{2}} ; \\
\frac{l}{R} & =\frac{1}{1.841} \tanh \left(\frac{1.841 h}{R}\right) ; \\
\alpha & =\frac{2 \delta}{\varepsilon^{2}}
\end{aligned}
$$

where $\delta$ is the damping ratio. For large fluid depth, $h / R>1.5$ ( $R$ is the tank radius), damping occurs predominantly in the side-wall Stokes boundary layers and is of the form 


$$
\delta=C_{1}\left(\frac{\nu^{2}}{R^{3} g}\right)^{1 / 4}
$$

where $\nu$ is the kinematic viscosity and $g$ is the gravity or axial acceleration. For the lowest asymmetric mode, $C_{1} \approx 1$ [8]. The small parameter $\varepsilon$ in Eqs. (1) and $(3)$ is

$$
\varepsilon=\left(1.684 \frac{A_{f}}{R}\right)^{1 / 3} .
$$

Theoretically, this parameter must be $\varepsilon \ll 1$, but experiments show [8] that the theory remains valid to leading order when $\varepsilon$ is not small but $\varepsilon<1$. The natural frequency of the lowest asymmetric mode is

$$
\omega_{1} \equiv \omega_{11}=\left[g k_{11}\left(1+\frac{\sigma k_{11}^{2}}{\rho g}\right) \tanh \left(k_{11} h\right)\right]^{1 / 2} .
$$

When the Bond number

$$
\mathrm{Bo}=\frac{\rho g R^{2}}{\sigma}
$$

is large, the surface tension term in the dispersion relation is negligible and when, in addition, $h / R>1.5$, the dispersion relation reduces to $\omega_{1}=\sqrt{g k_{1}}$, where the wave number $k_{1} \equiv k_{11}=1.841 / R$. It is of interest to point out that there is a critical nondimensional depth $h / R=0.506$ below which the nonlinearity changes from negative to positive and a depth $h / R=0.15$ below which harmonics dominate.

\subsection{Experimental Conditions}

The asymmetric sloshing experiments were conducted in circular cylindrical tanks, one of diameter $d=2 R=300 \pm 4 \mathrm{~mm}$ and $60 \mathrm{~cm}$ deep, made of plexiglas and the other of diameter $d=156 \pm 0.6 \mathrm{~mm}$ and $25 \mathrm{~cm}$ deep, made of Pyrex. The natural periods of the lowest asymmetric modes are, respectively, $T_{1}=2 \pi / \omega_{1}=572$ and $413 \mathrm{~ms}$. These tanks, filled with water or alcohol to the desired depth $h$, were mounted on an oscillating table. The fill ratio was always $h / R>1.2$ (generally, 1.5) satisfying deep-water conditions $\left(\tanh \left(k_{1} h\right)>0.976\right.$ in the dispersion relation). The Bond number on the tank scale is Bo $=\rho g R^{2} / \sigma>800$ so that surface tension effects are negligible. Only the sizes of drops and bubbles produced by breaking depend on surface tension. The containers were subjected to a horizontal displacement $x=A_{f} \sin \omega t$ in the range of $0.5 \leq f \leq 5 \mathrm{~Hz}$ and $0.05 \leq A_{f} \leq 5 \mathrm{~mm}$. The forcing frequency $f$ and forcing amplitude $A_{f}$ were measured to high accuracy with an optical displacement probe. The wave amplitude was measured with capacitance probes 
that were calibrated before and after each experiment and have a resolution of $0.2 \mathrm{~mm}$. These probes were positioned close to the tank wall and along a line parallel to the tank movement $\left(\theta=0^{\circ}\right.$, position $\left.P 1\right)$ and perpendicular $\left(\theta=90^{\circ}\right.$, position $\left.P 2\right)$ to the direction of the tank movement. The probe at $P 2$ indicated whether or not a swirling wave component was present. The shape of the liquid surface was obtained by visualizations using backlighting and image analysis.

\subsection{Results: Asymmetric Sloshing}

Figure 1 shows the wave amplitude response $b=\eta(R, \theta, n T)$, at $\theta=0^{\circ}(P 1)$ and $\theta=90^{\circ}(P 2)$ made dimensionless by the wavelength $\lambda \equiv \lambda_{1}=3.411 R$, as a function of dimensionless frequency $\omega / \omega_{1}$ for four forcing amplitudes $A_{f} / R$. All experimental points correspond to steady-state wave motions. The open symbols indicate planar waves and the closed symbols swirling waves. The amplitude response curves show clearly that the limits of existence of steady-state planar waves depend on both, the forcing frequency and forcing amplitude, that are combined in the frequency-offset parameter of Eq. (1). For the four dimen-

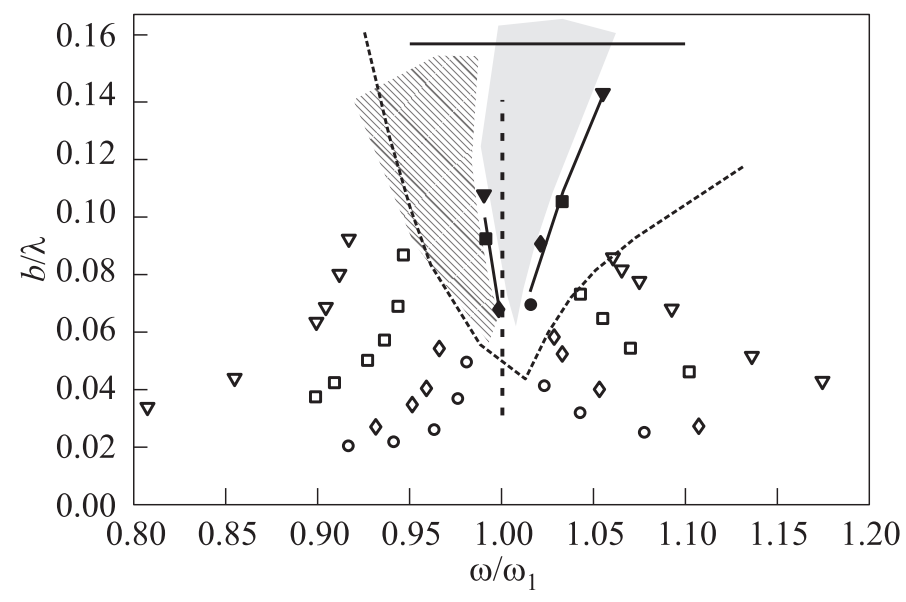

Figure 1 Amplitude-frequency diagram for four different forcing amplitudes $A_{f} / R$ : $\nabla$, planar wave mode $1 ; \mathbf{\nabla}$, swirling wave mode for $A_{f} / R=0.0266 ; \square, \mathbf{\square}, A_{f} / R$ $=0.0133 ; \diamond, \diamond, A_{f} / R=0.0066 ; \circ, \bullet, A_{f} / R=0.0033$. A stable swirling wave (shaded region) exists between the filled symbols and chaos is observed to the left of it (hatched region). The dotted branches are the bounds of swirl of Abramson [4]. The upper horizontal line indicates $b \omega^{2}=g$, giving $b / \lambda=0.16(\lambda=3.411 R)$. The tank, filled with water to $h / R \approx 1.5$, has a radius $R=78 \mathrm{~mm}$ 

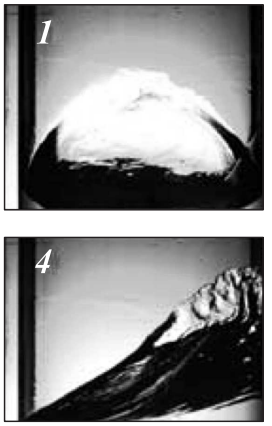
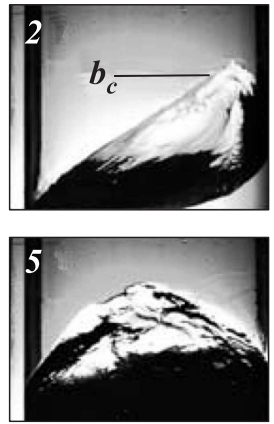
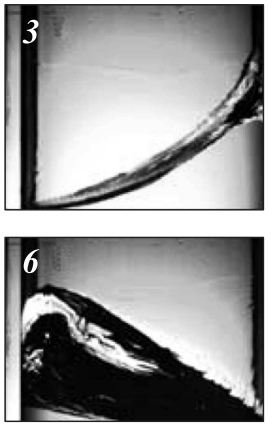

Figure 2 Images of swirling wave in circular cylinder of radius $R=150 \mathrm{~mm}$ partially filled with water. Views are in the direction normal to the tank motion. The time between two images is $67 \mathrm{~ms} ; T=2 \pi / \omega=570 \mathrm{~ms} ; h / R \approx 1.2 ; \omega / \omega_{1} \approx 1.02$, and $A_{f} / R=0.023$

sionless forcing amplitudes $A_{f} / R=0.0266,0.0133,0.0066$, and 0.0033 shown in Fig. 1, the parameter $\varepsilon$ given by Eq. (5) is $\varepsilon=0.356,0.282,0.224$, and 0.177, respectively. The damping parameter of Eq. (3) is $\alpha \approx 0.061,0.098,0.155$, and 0.248 , respectively, for the four forcing amplitudes and water (for alcohol the $\alpha$ values are about $20 \%$ larger). These values of the damping parameters are indicative of small damping where the resonance curves are qualitatively similar, exhibiting at least four bifurcation points when the depth parameter of Eq. (2), is constant ( $h / R$ large). Above the natural frequency, $\omega / \omega_{1}>1$, when for a given forcing amplitude, the forcing frequency is slowly decreased by small decrements, the planar wave amplitude increases (soft spring behavior) until a critical frequency is reached at which the motion bifurcates to a swirling wave; this bifurcation point is denoted as $\beta_{4}$ by Miles [1]. The same bifurcation to a swirling wave mode is obtained when the forcing frequency is fixed and the forcing amplitude is increased by small increment.

When starting at $\omega / \omega_{1}<1$ and then increasing the forcing frequency by small increments, the planar wave amplitude, for a given forcing amplitude, increases with frequency until the wave amplitude reaches a turning point (point $\beta_{3}$ in the Miles' theory [1]) and then grows rapidly until wave breaking occurs. This regime is referred to as chaos $[1,2]$. The upper horizontal line in Fig. 2 is the limit where the wave amplitude is such that the downward acceleration $b \omega^{2}$ is equal to gravity; this wave amplitude is referred to as $b_{c}$. Breaking requires $b \geq b_{c}[10]$. However, chaotic sloshing can occur without breaking that is without reaching amplitudes such that the downward wave crest acceleration equals that of gravity. The different domains of wave motions shown in Fig. 1 can also be represented in the form of a phase diagram, i.e., dimensionless forcing amplitude $A_{f} / R$ as a function of $\omega / \omega_{1}$. That writes [8]: 


$$
\frac{A_{f}}{R}=\frac{1}{1.684}\left[\frac{\left(\omega / \omega_{1}\right)^{2}-1}{\beta_{i}}\right]^{3 / 2}
$$

where $i=2,3, \ldots, 6$. The bounds are given by the specific values $\beta_{2}, \beta_{3}, \beta_{4}$, and $\beta_{5}$ (or $\beta_{6}$ ) calculated from the Miles' theory [1]. For $\alpha^{2} \ll 1$ and large fluid depth, $h / R>1.5$, the bifurcation point $\beta_{1}$ does not exist and $\beta_{2}=-0.36$ (bifurcation from swirl to chaos). The other bifurcation points are to leading order (higher order terms are in $\alpha^{2}$ and amount to less than $2 \%$ of the leading order terms) $\beta_{3}=-1.55, \beta_{4}=0.735, \beta_{5}=0.108 / \alpha^{2}$, and $\beta_{6}=0.717$. The bifurcation point $\beta_{5}$ is not physical. The values of these bifurcation points change with fluid depth when it is no longer asymptotically large, that is, when $h / R<1$. The values can be calculated from the Miles' theory but there is no experimental support, especially for cases when $h / R$ is in the neighborhood of the critical values $h / R=0.506$ and 0.15 . Now, the three main modes of sloshing motions will be discussed.

Steady state planar waves. Figure 1 indicates that the steady-state planar wave amplitude depends on the forcing amplitude and forcing frequency. Linear oscillators would suggest that

$$
\frac{b}{A_{f}} \approx \frac{2 K^{2}}{\left|1-K^{2}\right|}
$$

where $K=\omega / \omega_{1}$. While for $\omega / \omega_{1}<1$, the data would collapse reasonably well, this is not the case for $\omega / \omega_{1}>1$. The weakly nonlinear theory [1] predicts this asymmetry. The planar wave amplitude response curves terminate at the fixed points $\beta_{3}=-1.55$, and $\beta_{4}=0.735$, giving $\omega / \omega_{1}=\left(-1.55 \varepsilon^{2}+1\right)^{1 / 2}$ and $\omega / \omega_{1}=\left(0.735 \varepsilon^{2}+1\right)^{1 / 2}$, respectively.

Swirling wave. The swirling wave mode also referred by Ibrahim [3] to as rotary sloshing is observed when $\beta_{2}<\beta<\beta_{4}$. Figure 2 shows images of a large amplitude $(b>0.16 \lambda)$ swirling wave taken at 67 -millisecond interval that corresponds to 0.12 wave period. There are fairly large disturbances in the vicinity of the (flat-top) wave crest (local breaking) but the wave remains stable. Nonlinear waves can transfer angular momentum to the whole liquid column that starts to rotate $[12,11]$. The result is a Doppler shift, causing the wave frequency to increase with respect to the forcing frequency. Because of the liquid rotation, the swirl can be maintained up to fairly large forcing frequencies; here, up to $\omega / \omega_{1} \approx 1.3$, when the forcing frequency is increased by small increments [8]. There is continuous local wave breaking near the wave crest but as long as the forcing frequency remains below the collapse frequency, the swirl wave is maintained. When the forcing frequency is further increased by a small amount, the swirl wave suddenly collapses and the motion switches to a small amplitude, out of phase, planar wave motion.

Chaotic sloshing. Chaotic sloshing occurs when $\beta_{3}<\beta<\beta_{2}$. When the experiments are started at forcing frequencies below resonance and steady-state 


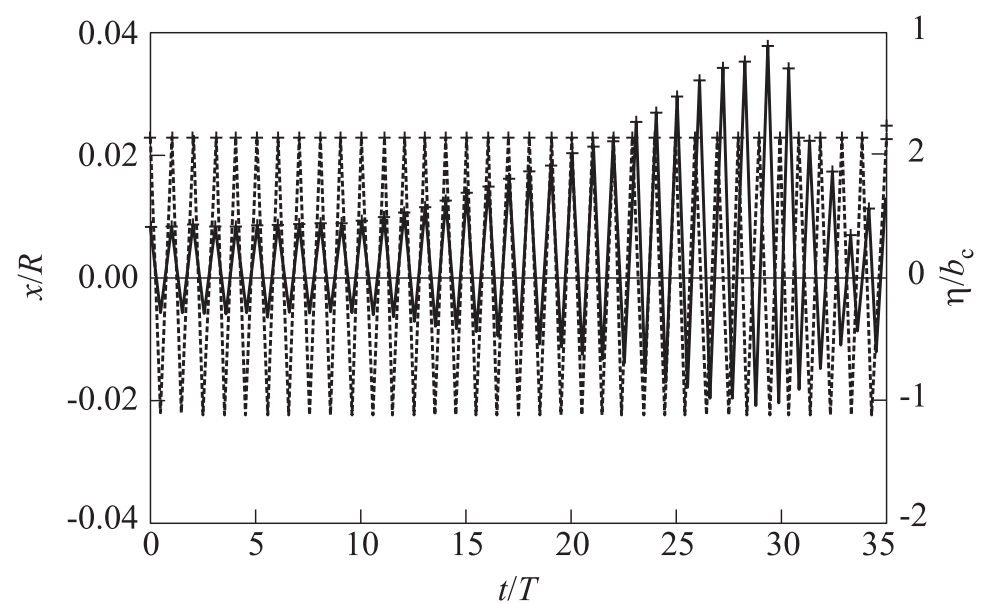

(a)

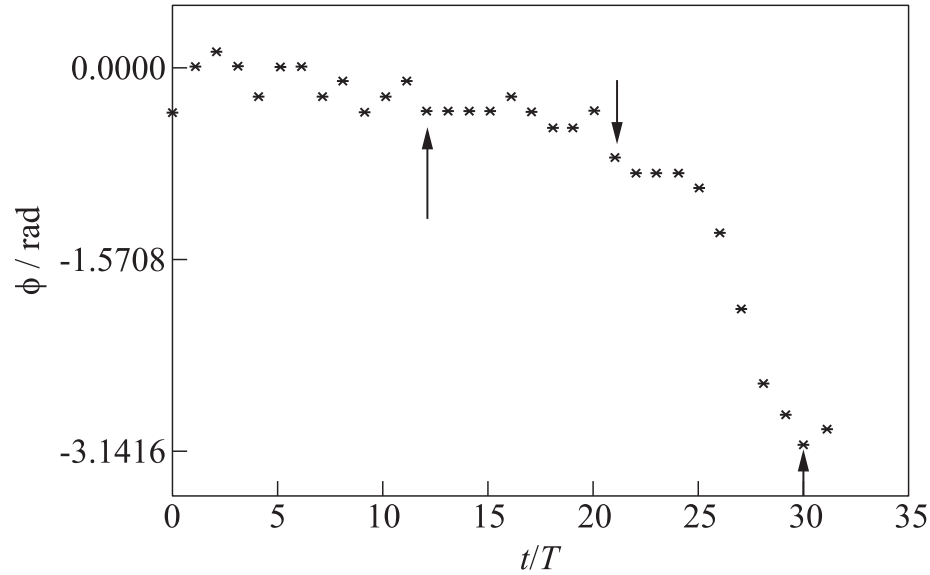

(b)

Figure 3 Time evolution of dimensionless wave amplitude $\eta / b_{c}$ vs. dimensionless time $(a)$ and corresponding phase lag $\phi(b)$. The forcing amplitude (left scale in $(a)$ ) is $A_{f} / R=0.022, R=150 \mathrm{~mm}, h / R=1.5$. Up to $t / T=10$, the forcing frequency was $\omega / \omega_{1}=0.89$, then it was increased to $\omega / \omega_{1}=0.92 . T=2 \pi / \omega=622$ and $640 \mathrm{~ms}$, $b_{c}=0.54 R$

planar wave motion is established, a small increase in forcing frequency leads to a rapid increase in wave amplitude if the turning point $\beta_{3}$ is crossed. Rapid increase in wave amplitude and wave breaking occurs in a similar way in the whole range $\beta_{3}<\beta<\beta_{2}$. The growth in wave amplitude near $\beta_{3}$ and wave breaking is illustrated in Fig. $3 a$ where the surface displacement $\eta$ as a function 
of time, measured with the capacitance probe located at point $P 1(\theta=0)$ is shown. The surface displacement is made dimensionless by $b_{c}$ and time by the forcing period $T=2 \pi / \omega$ (calculated with $\omega=0.89 \omega_{1}$ ). It is seen in Fig. $3 a$ that as the wave amplitude grows, the motion gets more and more asymmetric with the positive, maximum surface displacements reaching nearly twice the negative displacements. The motion is initially in phase with the forcing but then the phase lag increases with wave amplitude (Fig. $3 b$ ). When $b>b_{c}$, the phase lag increases rapidly to $-\pi / 2$ and is $-\pi$ at collapse. After breaking of the planar wave, an irregular swirl is generated and when the irregular sloshing motion has sufficiently decayed, the planar wave grows again in amplitude until breaking. This behavior occurs quasi-periodically. The growth of the planar wave amplitude is to first approximation linear in time (see Fig. $3 a$ ) and nearly proportional to the forcing amplitude in the form

$$
\frac{b}{R} \approx C_{2} \pi\left(\frac{A_{f}}{R}\right)^{n} \frac{t}{T} .
$$

Linear oscillator theory suggests $n=1$. Taking this value of $n$, the constant $C_{2}=0.3$ to 1.5 depending on initial conditions. In general, the growth rate was found to be larger when the wave amplitude in the chaotic regime grows from rest, whereas in the case of an established planar wave motion with a following small forcing frequency increase to the chaotic regime, the growth rate is less. The Miles theory [1] would suggest $n=2 / 3$ giving

$$
\frac{d(b / R)}{d \tau}=0.702 C_{2}
$$

where $\tau=\left(\varepsilon^{2} \omega t\right) / 2$ is the slow dimensionless time scale in the Miles' theory. For large wave amplitudes, the weakly nonlinear theory is no longer valid and the exponent may deviate from $n=2 / 3$. In the fully chaotic regime, wave breaking and collapse show a similar behavior except that further away from the swirl wave boundary, there is less or no regular swirl generated when the planar wave motion collapses. A layer moves up the wall and the crest is destabilized in the crosswise direction and two perturbations begin to grow at the edges of the layer. A period later, the pattern is repeated but is less regular. Before the violent wave destabilization (collapse), spilling occurs [8].

\section{IMPULSIVE FORCING AND AXISYMMETRIC SLOSHING}

An example of impulsive forcing, after a short microgravity phase, is the falling coffee cup experiment by Milgram [13]. Membranes, simulating high surface 
tension, have also been used [9] to obtain small initial Bond numbers simulating a microgravity phase. Rupture of the membrane leads to a sudden step increase in Bond number. It is also possible to use a rigid barrier instead of a membrane. The sudden withdrawal of the barrier (infinite surface tension) is analogous to a sudden increase in acceleration after low gravity conditions, corresponding to a sudden step increase in Bond number. These experiments are referred to as axisymmetric dam-break experiments.

\subsection{Experimental Conditions}

Figure 4 shows a schematic view of the dam-break experiments. The container, made of glass, has an inner diameter $2 R$ $=296 \pm 2 \mathrm{~mm}$ and depth $60 \mathrm{~cm}$; the error in verticality is $0.4^{\circ}$. The initial liquid column conditions are achieved by positioning a barrier (inner tube) of outer radius $R_{1}=10 \mathrm{~cm}$ and $4 \mathrm{~mm}$ wall thickness at the center of the container. This tube is open on both ends and has a watertight contact with the tank bottom. The annular space of $d=R-R_{1}=48 \mathrm{~mm}$ is filled with liquid (water or alcohol) to depth $h_{1}$ and the central space to depth $h_{2}$. The important scale is $\Delta h=h_{1}-h_{2}$. The three experimental conditions considered are: $\operatorname{Exp} 1-h_{1}=15 \mathrm{~cm}, h_{2}=0$; $\operatorname{Exp} 2-h_{1}=5 \mathrm{~cm}, h_{2}=0 ; \operatorname{Exp3}-$ $h_{1}=10 \mathrm{~cm}, h_{2}=5 \mathrm{~cm}$. The experiments begin by removing suddenly the barrier (axisymmetric barrier) of radius $R_{1}$. It is crucial that the barrier be removed very rapidly, here with an acceleration of

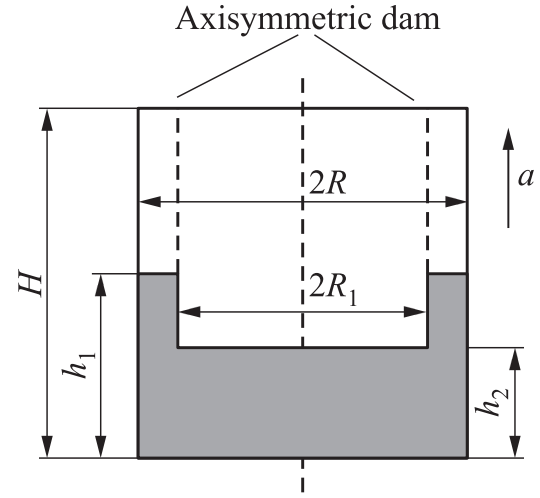

Figure 4 Schematic representation of the dam-break experiments. The initial state of the liquid, indicated by the shaded area, is maintained by the axisymmetric barrier of outer radius $R_{1}$ in the container of radius $R$. The step change $\Delta h=h_{1}-h_{2}$ of the free surface simulates the rise of the liquid near the container wall under low gravity conditions about $10 \mathrm{~g}$, so that the fluid column has negligible motion during barrier withdrawal. The flow was determined by image processing. The images were taken with a high speed camera at $500 \mathrm{~Hz}$ and a resolution of $0.322 \mathrm{~mm}$ per pixel for a $1536 \times 1024$ pixel matrix of the image.

\subsection{Results: Geyser Formation and Axisymmetric Sloshing}

In Fig. 5, geyser initiation (right after the liquid front arrived at the center) and maximum geyser heights and shapes are shown for the three experimental 


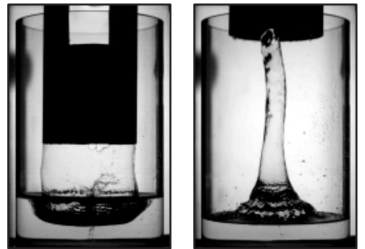

(a)

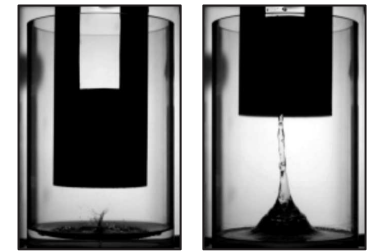

(b)

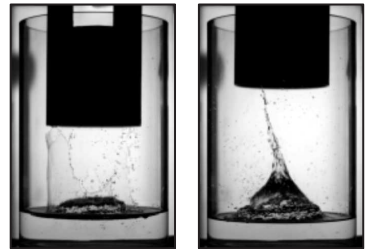

(c)

Figure 5 Geyser initiation and shape: $(a) h_{1}=\Delta h=15 \mathrm{~cm}(\mathbf{E x p 1}), t_{1}=0.05 \mathrm{~s}$ (initiation) and $t_{2}=0.45 \mathrm{~s}$ (maximum height of geyser); (b) $h_{1}=\Delta h=5 \mathrm{~cm}(\mathbf{E x p 2})$, $t_{1}=0.13 \mathrm{~s}$ and $t_{2}=0.51 \mathrm{~s} ;(c) h_{1}=10 \mathrm{~cm}, h_{2}=5 \mathrm{~cm}, \Delta h=5 \mathrm{~cm}(\operatorname{Exp} 3), t_{1}=0.14 \mathrm{~s}$ and $t_{2}=0.41$ s. $t_{l}=\sqrt{2 \Delta h / g}=0.175 \mathrm{~s}$ in Exp1 and $0.101 \mathrm{~s}$ in Exp2 and Exp3. The scale in the images is given by the tube diameter equal to $20 \mathrm{~cm}$

conditions. The first observation is that the maximum geyser height $h_{g \mathrm{~m}}$ is considerably larger than $\Delta h$. The maximum height is given by

$$
h_{g \mathrm{~m}}=\frac{U_{g 0}^{2}}{2 g}+h_{2}
$$

where $h_{g \mathrm{~m}} / \Delta h \cong 5.3$ in $\operatorname{Exp1}, h_{g \mathrm{~m}} / \Delta h \cong 14$ in $\operatorname{Exp2}$, and $h_{g \mathrm{~m}} / \Delta h \cong 9.4$ in Exp3. The overall conservation of mass and momentum would support the existence of a nearly cylindrical geyser of height equal to $\Delta h$ (except for some overshoot) and of radius

$$
R_{g} \cong \sqrt{2 R d}\left(1-\frac{d}{4 R}\right) .
$$

The radius of the geysers at the base, shown in Fig. 5, is close to this value but the shape is conical with the diameter decreasing rapidly with height, and then it remains nearly constant at a value much less than $R_{g}$. The maximum geyser height corresponds to an initial geyser velocity $U_{g 0}=\sqrt{2 g h_{g \mathrm{~m}}}$ that is much larger (by the factor $\sqrt{h_{g \mathrm{~m}} / \Delta h}$ ) than the maximum liquid column velocity $U_{1}=\sqrt{2 g \Delta h}$. Momentum is transferred to only a small fraction of the liquid mass that is projected up to height $h_{g \mathrm{~m}}$. When this geyser collapses, considerable gas entrainment into the liquid occurs (see Fig. 7).

The geyser diameter $r_{g}$ above the conical base depends on the thickness of the front of the liquid layer when it arrives at the center. In Fig. $5 b$, the liquid front remains thin and $r_{g}$ is small but is one order of magnitude larger than the capillary scale $l_{c}=\sqrt{\sigma /(\rho g)}$ at arrival. In Fig. $5 a$, the front is much thicker. The reason for this is that the ratio of front acceleration time, equal to the time of collapse of the liquid column $t_{l}=\sqrt{2 \Delta h / g}$, to radial propagation time $t_{R}=\sqrt{2 R_{1}^{2} /(g \Delta h)}$ is 1.5 in Exp1 and 0.5 in Exp2. Figure 5 indicates that 


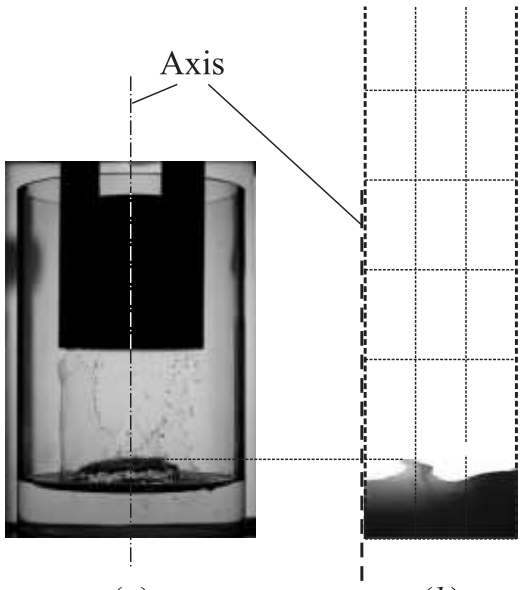

(a)

(b)

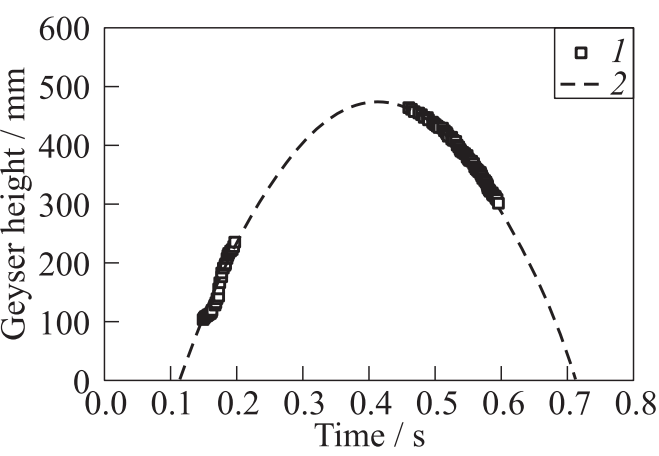

(c)

Figure 6 Geyser evolution in Exp3: (a) image just before geyser appearance; (b) numerical simulations (J.-P. Vila, ONERA) showing the flip-through; and (c) geyser height as a function of time: 1 - experiment with alcohol and 2 - parabolic fit

the geyser height depends principally on $\Delta h$ and, of course, on the acceleration applied to the container, that is, $g$ in the present experiments (in Migram's experiments [13], it is $d V / d t$ where $V$ is the container velocity at impact). The geyser evolution (its height and diameter) in Fig. $5 c$ is close to that of Fig. $5 b$. However, the geyser initiation is different.

This initiation is clearly demonstrated by numerical simulations shown in Fig. $6 c$ (curtsey of Jean-Paul Vila, ONERA). When the initial step change in interface height is suddenly released, a surface wave starts to propagate toward the center and an annular front forms (Fig. 6b) that accelerates and merges at the center. This is referred to as flip-through (private communications D. H. Peregrine) also encountered when a surface wave approaches a wall. In the experiments (Fig. 6a), only the envelope of this front is seen.

After collapse of the geyser, a second geyser of smaller height emerges and then, the height of the following geysers decreases until the liquid sloshing motion has been damped completely at time $t_{5}$. This sloshing motion consists of a symmetric, nonlinear wave mode as shown in Fig. 7 for conditions of Exp3 and for water. For alcohol, the sloshing motion is identical to that of water. However, the larger Bond number (lower surface tension of alcohol) gives rise to smaller drops associated with breaking of the geyser tip and smaller bubbles as gas is entrained on geyser collapse. The relevant Weber number is

$$
\mathrm{We}=\frac{\rho U_{g 0}^{2} r_{g}}{\sigma}
$$



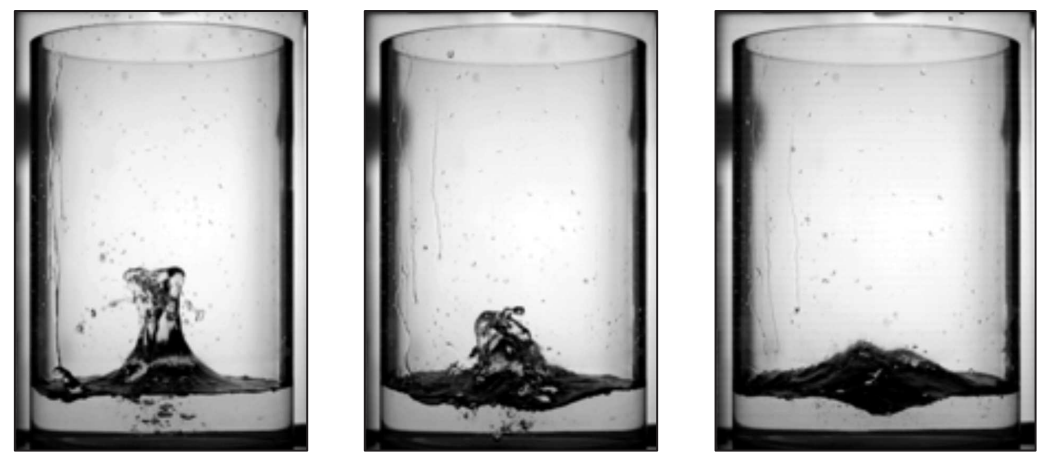

Figure 7 Decay of sloshing motion in Exp3 with water. The images are taken at 400-millisecond interval

which gives together with

$$
U_{g 0}=\sqrt{\frac{h_{g m}}{\Delta h}} U_{1}
$$

and

$$
r_{g} \sim \frac{R_{g} \Delta h}{R}
$$

a characteristic Bond number

$$
\mathrm{Bo}=\frac{\rho g \Delta h^{2}}{\sigma} \sqrt{\frac{d}{R}} \frac{h_{g \mathrm{~m}}}{\Delta h} .
$$

In all the experiments, this Bond number is $>10^{3}$. The alcohol experiments have a larger Bond number and the second, third, and subsequent geysers are more irregular; drops are smaller and more gas is entrained or at least it remains in the liquid layer for a longer time. The time interval between the images shown in Fig. 7 is close to $T \approx 400 \mathrm{~ms}$ that corresponds to a frequency $\omega$ $=2 \pi / T \approx 15.7 \mathrm{rad} / \mathrm{s}$. The dispersion relation

$$
\omega_{01}^{2}=k_{01} g \tanh \left(k_{01} h_{l}\right)
$$

where $h_{l}$ is the fluid layer depth and $k_{01}=3.83 / R$ is the wave number for the first symmetric mode, gives $\omega_{01}=15.5 \mathrm{rad} / \mathrm{s}$. The decay of the sloshing motion is exponential, of the form $A=A_{0} \exp (-\delta \omega t)$, where $A_{0}$ is the initial amplitude. The initial decay of geyser amplitude is large, $\delta \approx 0.023$, because the liquid layer is very agitated (probably turbulent). Then, the decay is considerably slower, $\delta$ $\approx 0.0073$. Dissipation is at the boundaries of the container (side and bottom) 
and also internal in the beginning and then only at the boundaries. The damping ratio $\delta$, in accordance with Eq. (4), is given by

$$
\delta=C_{2}\left(\frac{\nu^{2}}{g R^{3}}\right)^{1 / 4}
$$

with the constant $C_{2}$ being about 16 in the beginning and 3 for the decay after the second or third geyser.

\section{CONCLUDING REMARKS}

Asymmetric sloshing is controlled by three nondimensional (similarity) parameters that are the liquid depth parameter, the frequency offset (depending on forcing frequency and forcing amplitude), and the damping parameter in agreement with the Miles theory [1]. This theory contains a small parameter, $\varepsilon \ll 1$. Experiments show that the theory remains valid to leading order when $\varepsilon$ is not small but $<1(\varepsilon \leq 0.5)$. The bounds of existence of the different sloshing regimes are clearly established, namely, planar, swirling, and breaking waves. In the chaotic regime, wave breaking occurs quasi-periodically with growth of planar wave amplitude, collapse followed by irregular swirl, and again growth of planar wave amplitude. Surface tension effects on the tank scale are assumed to be negligible (large Bond number Bo $=\rho g R^{2} / \sigma$ ). However, surface tension effects are important in the final stages of wave breaking (drop and bubble formation).

The results of the dam-break experiments show that when a relatively small step change in surface height is suddenly subjected to an axial acceleration, a high velocity geyser emerges, followed by violent sloshing with gas entrainment. This situation is encountered on engine restart after a microgravity phase.

\section{ACKNOWLEDGMENTS}

This work was financially supported by contracts CNES No. 5866 and No. 1616 within the French-German program COMPERE.

\section{REFERENCES}

1. Miles, J. W. 1984. Resonantly forced surface waves in a circular cylinder. J. Fluid Mech. 149:15-31.

2. Funakoshi, M., and S. Inoue. 1988. Surface waves due to resonant horizontal oscillations. J. Fluid Mech. 192:219-47. 
3. Ibrahim, R. A. 2005. Liquid sloshing dynamics. Cambridge University Press.

4. Abramson, H.N. 1966. The dynamic behavior of liquids in moving containers. NASA Technical Report SP-106.

5. Miles, J. W. 1984. Internally resonant surface waves in a circular cylinder. J. Fluid Mech. 149:1-14.

6. Waterhouse, D. D. 1994. Resonant sloshing near a critical depth. J. Fluid Mech. 281:313-18.

7. Faltinsen, O.M., O.F. Rognebakke, and A.N. Timokha. 2003. Resonant threedimensional nonlinear sloshing in a square-base basin. J. Fluid Mech. 487:1-42.

8. Royon-Lebeaud, A., E. J. Hopfinger, and A. Cartellier. 2007. Liquid sloshing and wave breaking in circular and square-base cylindrical containers. J. Fluid Mech. 577:467-94.

9. Baumbach, V., E. J. Hopfinger, and A. Cartellier. 2005. The transient behavior of a large bubble in a vertical tube. J. Fluid Mech. 524:131-42.

10. Taylor, G. I. 1953. An experimental study of standing waves. Phil. Trans. Roy. Soc. CCXVIII:44-59.

11. Prandtl, L. 1949. Erzeugung von Zirkulationen beim Schutten von Gefässen. Z Angew. Math Mech. 29:8-9.

12. Faller, A. J. 2001. The constant- $V$ vortex. J. Fluid Mech. 434:167-80.

13. Milgram, J.H. 1969. The motion of a fluid in a cylindrical container with a free surface following vertical impact. J. Fluid Mech. 37:435-48. 